\title{
Control of static and dynamic hydrophobicity of solid surface and its application
}

\author{
Akira NAKAJIMA ${ }^{\dagger}$ \\ Department of Metallurgy and Ceramic Science, Graduate School of Science and Engineering, \\ Tokyo Institute of Technology, 2-12-1 Ookayama, Meguro-ku, Tokyo 152-8552
}

Technologies of hydrophobic coating on solid surface are important for various industrial items to impart functions such as antibeading, anti-rusting, anti-snow adhesion, self-cleaning, and lubrication. In recent, the demands of these functions are upgrading, and further improvements of performance are necessary. To meet today's requirements, detailed understandings on the relation between solid characteristics (such as structure, chemical composition, and their arrangement) and static/dynamic hydrophobicity is indispensable. So far, the research on this relation has been conducted in the part of fluid mechanics, tribology, and surface chemistry. However, recent researches revealed that static and dynamic liquid droplet behaviors are affected by nano-scale surface characteristics on hydrophobic solids, and the importance of the viewpoint from materials science is gradually increasing in this topic. This paper presents a review of recent studies on the relation between solid characteristics and static/ dynamic hydrophobicity, mainly of the past decade of our group.

(2011 The Ceramic Society of Japan. All rights reserved.

Key-words : Hydrophobicity, Wettability, Contact angle, Sliding angle, Sliding acceleration

[Received July 6, 2011; Accepted August 9, 2011]

\section{Introduction}

Wetting of solid surface by liquid has been a research topic from fundamental to technological aspects through ages, because it can lead various physical or chemical phenomena between solid and liquid to helpful direction. The phenomenon of wetting is, in other words, the adhesion of liquid on solid surface. The nature of wettability is more complex than solid surface because it is related to three phases: solid, liquid, and gas. Moreover, various factors affect wettability of a solid surface. When one character of a solid surface (such as roughness) is altered, other characters such as the surface topology or its chemical composition often change in conjunction with the character altered. Thus, it is commonly difficult to discuss the results of surface wettability obtained from experiments with retaining academic rigor. Since the main research interest for industrial materials is the behavior of millimeter-size droplets on a solid surface with more than a few centimeters, comparison of wettability among samples whose one surface character differs greatly is often conducted with ignoring the contribution of other characteristics. Thanks to the advance of probe microscopy and optics, investigations on nano-scale roughness or chemical heterogeneity become feasible in recent. Then it is revealed that even such nano-scale heterogeneity affects static and dynamic wettability in macroscopic scale.

Hydrophobic coatings are quite important for wettability control. They are expected for various industrial uses such as anti-wetting, anti-snow (or ice)-adhesion, anti-rusting, and reduced friction resistance by decreasing solid-liquid interaction. General image of a hydrophobic surface is one on which water forms a round droplet that is easily removed. However, the expected properties cannot always be obtained unless precise design and process control of the solid surface are conducted.

$\uparrow$ Corresponding author: A. Nakajima; E-mail: anakajim@ceram. titech.ac.jp
This report presents results of recent studies on the relation between solid characteristics and static/dynamic hydrophobicity from the viewpoint of materials science.

\section{Static and dynamic wettability on solid surface}

\subsection{Static wettability}

Wettability of solid surface is commonly compared by the contact angle $(\theta)$ given by Young's equation as

$$
\gamma_{\mathrm{sv}}=\gamma_{\mathrm{sl}}+\gamma_{\mathrm{lv}} \cos \theta
$$

where $\gamma_{\mathrm{sl}}, \gamma_{\mathrm{sv}}$, and $\gamma_{\mathrm{lv}}$ respectively represent the interfacial free energies per unit area of solid-liquid, solid-gas, and liquid-gas interface. Since energy is scalar amount, these energies do not include the concept of direction. When we consider directionality, these three amounts are treated as vector amount, namely surface tension for each two phases per unit length at the three-phase (solid, liquid, and gas) contact line. In both cases, the unit becomes equivalent because $\mathrm{J} / \mathrm{m}^{2}$ is equal to $\mathrm{N} / \mathrm{m}$. The surface with a great contact angle is hydrophobic. Wettability of the solid surface by the liquid decreases concomitantly with increasing the contact angle. It is noteworthy that the part which determines the contact angle is fundamentally, not the entire contact area, but in the vicinity at the three-phase contact line.

Although the atom in solid is surrounded by other atoms or ions, an atom at the surface has a deficiency of one part of surrounding atoms. Therefore, entire interactions from other atoms or ions at the surface atom are different from that in a bulk solid, and this is the qualitative origin of the surface or interface energy. Since surface energy is defined as a Gibbs free energy per unit area, ${ }^{1)}$ it depends on the temperature.

According to theories of Dupre and Girifalco-Good in their work on adhesion, ${ }^{2)}$ the contact angle is described as follows.

$$
\cos \theta=\left\{2 \phi \sqrt{\left(\gamma_{\mathrm{sv}} / \gamma_{\mathrm{lv}}\right)}\right\}-1
$$

Therein, $\phi$ is a correction factor, and its value is commonly around unity. This equation means that a decrease of $\gamma_{\mathrm{sv}}$ increases 
contact angle. By substituting $\phi$ and the surface energy of water as unity and $72.8 \mathrm{~mJ} / \mathrm{m}^{2}$, respectively, the maximum contact angle on a flat and smooth surface attainable merely by lowering the surface energy (the lowest surface energy value yet recorded is ca. $\left.6 \mathrm{~mJ} / \mathrm{m}^{2}, 3\right)$ as obtained from a surface with regularly aligned closest-packed- $\mathrm{CF}_{3}$ group) is ca. $115.2^{\circ}$.

Since three different phases merge at the three-phase contact line, an additional force which relates three phases exists on the line in addition to interface tensions between each two phases. This force is called as line tension, and described as $\sigma$ in the following equation.

$$
\gamma_{\mathrm{sv}}=\gamma_{\mathrm{sl}}+\gamma_{\mathrm{lv}} \cos \theta+\sigma / r
$$

where $r$ is the radius of the solid-liquid contact area. The values of line tension and other interfacial free energies are constant in a solid-liquid system. Therefore, a linear relation is obtainable between $\cos \theta$ and $1 / r$. By measuring contact angles with different masses of the liquid droplet and plotting $\cos \theta$ and $1 / r$, we can obtain the line tension value from the slope $\left(=-\sigma / \gamma_{\mathrm{lv}}\right)$. The reported value of the line tension measured using this method ranges from $10^{-9}$ to $10^{-5} \mathrm{~J} / \mathrm{m},{ }^{4)-7)}$ and recent researches revealed that hydrostatic pressure from the liquid plays an important role on this measurement. ${ }^{8), 9)}$ This value is fundamentally quite small compared to other interfacial free energies. Therefore, the effect of this tension is generally ignorable for the droplet with a common size such as $1-2 \mathrm{~mm}$ (In this case, Eq. (3) is equal to Young's equation). When the droplet size decreases to nano-liter scale, it affects contact angle. ${ }^{10)}$

\subsection{The limit of static wettability}

The sliding angle is a critical angle at which a droplet starts sliding down an inclined surface by gradual tilting, and contact angle at the advancing and receding edges of the droplet are called as the advancing and receding contact angles (Fig. 1). They are the criteria for the limitation of interface energy balance at the three-phase contact line and depend on the droplet mass. Furmidge derived an equation that describes the relation between the sliding angle and receding and advancing contact angles as follows. ${ }^{11)}$

$$
\frac{m g \sin \alpha}{w}=\gamma_{\mathrm{lv}}\left(\cos \theta_{\mathrm{R}}-\cos \theta_{\mathrm{A}}\right)
$$

Therein, $\alpha, m, g, w$, and $\theta_{\mathrm{R}}$ and $\theta_{\mathrm{A}}$ respectively signify the sliding angle, the mass of the droplet, gravitational acceleration, the droplet width, and the receding and advancing contact angles. Since the receding and advancing contact angles are the critical angles at which a droplet can stay on a surface without sliding on an inclined surface by its own shape deformation, the sliding angle of the droplet is small when the difference of these angles is small. The direct difference (or the difference of cosine) of

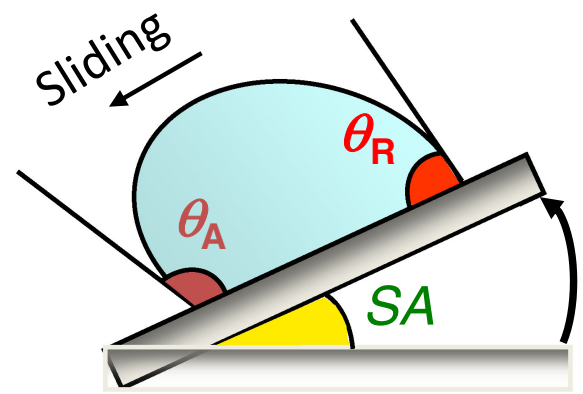

Fig. 1. (Color online) Schematic illustration of the sliding angle (SA) and the advancing $\left(\theta_{\mathrm{A}}\right)$ and receding $\left(\theta_{\mathrm{R}}\right)$ contact angles. advancing and receding contact angles are commonly called as contact angle hysteresis. Although modified relations have been proposed on the relation between the sliding angle and the receding and advancing contact angles, ${ }^{12), 13)}$ the fundamental relation among these three is the same as Eq. (4). Decrease of contact angle hysteresis by the removal of its origins is an effective approach for the design of surfaces with a small sliding angle.

Because sliding angle does not a function of time, tilting of solid surface should be carried out gradually for the measurement. The values of advancing and receding contact angles can be measured not only by droplet sliding but also by the expanding and contracting of water droplets through injection and suction of water to a droplet placed on a flat surface. Even for this measurement, injection and suction of water should be performed with a slow rate to ignore their rate dependence on advancing and receding contact angles. ${ }^{14)}$

\subsection{Dynamic wettability}

Although the sliding angle relates the motion or deformation of droplets, it is not an index of dynamic hydrophobicity such as sliding acceleration or velocity. Both the contact angle and the sliding angle are kind of thermodynamic properties because they do not include "time" as a parameter. Information about how fast the droplet can be removed from the surface at a certain tilt angle is often becomes more important than that on the lowest tilt angle at which the droplet slides down for the design of surface with excellent water shedding performance. Moreover, dynamic hydrophobicity relates directly to energy saving and environmentally friendly technologies by decreasing the friction drag between liquids and solids. However, understanding of the solid surface characteristics contributing to the dynamic hydrophobicity is insufficient.

Recently, studies of dynamic wettability have been stimulated by the availability of high-speed photography, advancement of motion-image sequence analysis, and rapid computation. These technologies facilitate the advancement of studies not only of sliding behavior but also on other ones such as bouncing, evaporation, and freezing.

Recent researches revealed that slipping and rolling motions exist in the mode of sliding for a water droplet. ${ }^{15)-17)}$ An effective approach for the direct observation of droplet's internal fluidity during moving is particle image velocimetry (PIV, Fig. 2). ${ }^{18), 19)}$ Using this technique, internal fluidity and velocity distribution during sliding are directly obtainable by tracking particles or their concentration dispersed into water. Studies using this system revealed that the contribution of the slipping mode is large on a surface with excellent dynamic hydrophobicity; the rolling/ slipping ratio depends on not only the solid surface but also measurement condition such as droplet mass and liquid viscosity. ${ }^{20), 21)}$ Therefore, comparison of dynamic hydrophobicity of solid surface should be performed on the experimental results obtained under the same measurement condition. Dynamic hydrophobicity is not water-spreading kinetics, but water-shedding properties from the surface. The evaluation of dynamic hydrophobicity on a surface with a low hydrophobicity requires special care.

\section{Factors for the wettability of solid}

\subsection{Surface chemical composition}

Recently, we prepared highly smooth $(R \mathrm{a}<0.2 \mathrm{~nm})$ coatings using various linear silanes, and evaluated their static and dynamic hydrophobicity. Both contact angle and sliding accel- 

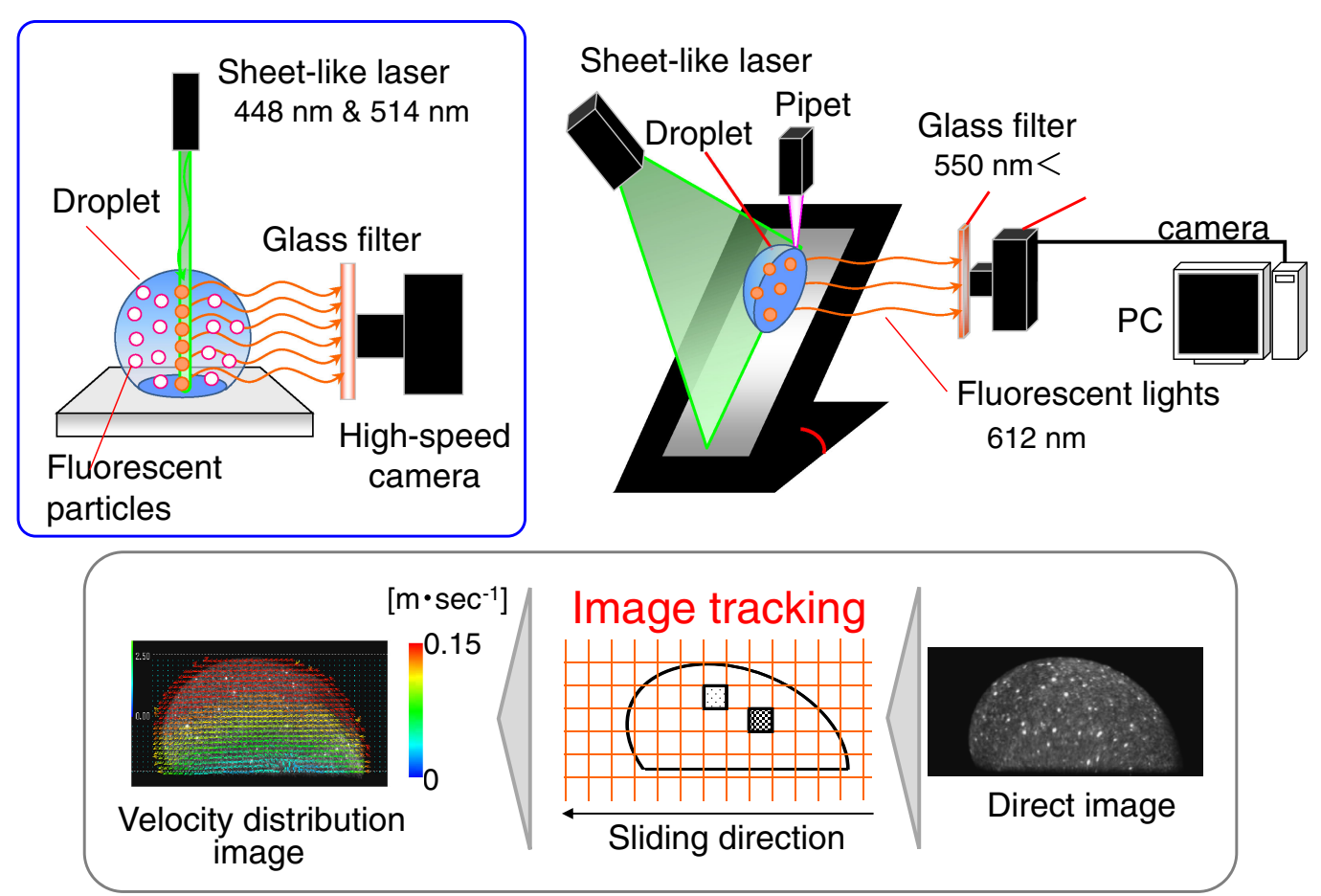

Fig. 2. (Color online) Observation of internal liquid fluidity by particle image velocimetry. Fluorescent particles are mixed with water. The particles emit light as a sheet-shaped laser irradiated perpendicular to the droplet. The image of the droplet sliding with light emission is recorded using a high-speed camera. The intensity and dispersion of the light in each pixel of the droplet image was tracked in a short time period and the velocity vector distribution in the image was calculated.

eration increased with increasing carbon number in the molecule till 6-8, then they are saturated. This result suggests that the chain length required for the fully-covered surface is around C6-C8. However, the sliding angle was independent to carbon numbers both for fluorocarbon or hydrocarbon moieties and was almost constant around $10^{\circ}$, which imply that sliding angle mainly governed by the surface homogeneity. ${ }^{22)}$ When the main subject is water droplets, both hydrocarbon-based silanes and fluorocarbon-based ones provide large contact angles, because their surface energy is enough smaller than that of water. However, the advantage of fluorocarbon-based ones increases when the main subject is the droplets of liquids such as organic solvent or oil.

Whereas silane coupling agents are commonly employed for hydrophobic treatments on inorganic solid surface, polymers are employed for the items of organic materials. By using glass transition behavior, smooth surface of polymer coating is effectively obtainable. Yoshida et al. prepared various smooth $(R \mathrm{a}=$ ca. $0.3-0.5 \mathrm{~nm})$ fluoromethacrylate (FMA)-methylmethacrylate (MMA) polymer coatings, and investigated the contact angle, sliding angle and sliding acceleration. ${ }^{23)}$ Both the contact angle and the sliding acceleration on the coatings increased concomitantly with an increased number of fluorocarbons in FMAs like silanes. However, the contact angle hysteresis shows a different trend. In the longer and shorter fluoroalkyl chains (FMA-F17, F19, and F3), the contact angle hysteresis decreased (=decrease in sliding angle) with increasing FMA concentration, although it increased in the medium-length chain (FMA-F9). They attributed this result to the aggregation effect of the longer fluoroalkyl chain and the larger reorientation of the medium chain at the water-polymer interface. Reorientation of the short chain might be slight because of its orientation freedom. Their results suggest that the sliding angle and sliding acceleration are governed by different factors in these coatings.

\subsection{Alignment or heterogeneity of surface energy}

Unless the size of a water droplet is more than its capillary length, the droplet forms spherical cap morphology on a hydrophobic surface. When surface energy is not homogeneous in the solid, the three-phase contact line changes from circle to different shape, and both static and dynamic wettability exhibit anisotropy with following alignment or heterogeneity of surface energy. ${ }^{24), 25)}$ The hydrophilic portions in a hydrophobic surface commonly inhibit the motion of the three-phase contact line (This phenomenon is well-known as pinning effect.), and lead the increase of sliding angle or the decrease of sliding acceleration. ${ }^{26)}$ Very recently, Furuta et al. prepared four patterned surfaces with hydrophilic areas of different sizes using photolithography with a smooth hydrophobic silane coating, and evaluated static and dynamic hydrophobicity. They demonstrated that the sliding angle and contact angle hysteresis of the water droplets for these surfaces increase concomitantly with increasing pattern size, suggesting that resistance for the sliding down of water droplets from a resting state is positively correlated with the pattern distance. However, the sliding velocity against pattern size decreased concomitantly with increasing pattern size. They deduced the interface between hydrophobic and hydrophilic regions play an important role on this result. Chemical surface defects provide different size dependence for sliding behavior between the critical moment at which a droplet slides down and the period when a droplet is sliding. ${ }^{27)}$

Suzuki prepared smooth fluoroalkylsilane coatings $\left(R_{\mathrm{a}}<\right.$ $0.2 \mathrm{~nm}$ ) with different surface coverage ratios on a Si substrate by changing the deposition time. Then the internal fluidity of water droplets sliding on the coatings was evaluated using PIV. ${ }^{28)}$ The sliding acceleration of the advancing contact line decreased concomitantly with the decreasing surface coverage ratio. However, the slipping/rolling ratio to the overall sliding 
acceleration was almost constant against surface coverage ratio. This result suggests that internal fluidity does not strongly affected by average surface chemical composition (surface coverage ratio of silane), when the physical roughness is small and silane distribution in the surface is homogeneous (surface composition is not segregated). They also prepared fluoroalkylsilane coatings with different physical and chemical heterogeneity on $\mathrm{Si}$ substrates by changing process condition, and demonstrated that sliding acceleration and its behavior (rolling/ slipping ratio during sliding) are affected to a remarkable degree by nano-scale structural and chemical heterogeneity. ${ }^{29)}$ This result means that the motion of the three-phase contact line is inhibited once structural and chemical heterogeneity exists in a solid surface simultaneously even when they are in nano-scale. Such surface affects not only the sliding of droplet, but also on the freezing ${ }^{30), 31)}$ and evaporation ${ }^{32)}$ behavior of water droplets.

Chen et al. proposed the following origins for contact angle hysteresis from the adhesion behavior of droplets on the mica surface coated with surfactants: (i) increasing the freedom of the surface molecules to reorder, (ii) increasing the load and time allowed for surfaces to remain in contact, and (iii) increasing the rate of separation or retraction. ${ }^{33)}$ However, the respective and combined contributions of these factors to practical contact angle hysteresis remain unclear. Their contribution is expected to differ among materials.

The chemical heterogeneity does not affect contact angle remarkably unless the heterogeneous part exists on the threephase contact line. ${ }^{34)}$ Marmur et al. pointed out that the Wenzel and Cassie equations (Eqs. (5) and (6), they will be described later. Cassie's equation commonly applied to the surface with two different surface energies.) are not expected to be valid when the droplet size approaches the order of magnitude to the roughness or chemical heterogeneity. ${ }^{35)}$ The effect of surface energy alignment to static and dynamic hydrophobicity is complex and depends on various factors such as chemical composition, size, amount, shape, and distribution of the portion of different surface energy.

\subsection{Surface roughness}

When roughness is imparted to a solid surface, its wettability is changed. Wenzel modified Young's equation and described contact angle $\theta^{\prime}$ on a rough surface as follows. ${ }^{36)}$

$$
\cos \theta^{\prime}=r\left(\gamma_{\mathrm{sv}}-\gamma_{\mathrm{sl}}\right) / \gamma_{\mathrm{lv}}=r * \cos \theta
$$

In those equations, $\theta$ denotes the contact angle on the smooth solid surface. In addition, $r$ signifies the roughness factor, which is defined as the ratio of the actual area of a rough surface to the geometrically projected area. This $r$ differs from the value of the arithmetic mean deviation of the roughness profile $(R \mathrm{a})$, which is commonly employed in the engineering field. In this equation, $r$ is always greater than unity. Therefore, the surface roughness enhances wettability of the solid surface (Hydrophilic surface becomes more hydrophilic, and hydrophobic surface becomes more hydrophobic.). Since the absolute value of $\cos \theta^{\prime}$ should be less than unity, this equation is applicable when $r$ is relatively small.

Cassie proposed an equation for a hydrophobic surface with large roughness. With increasing surface roughness, air intrudes into the hydrophobic solid-liquid interface. It is assumed that the interface comprises solid and air. When the unit area of the surface has a wetted solid surface area fraction $f$ with a water contact angle $\theta$, the contact angle on the surface can be expressed as the following equation, assuming a $180^{\circ}$ water contact angle for air. ${ }^{37)}$ (a) Young's Equation

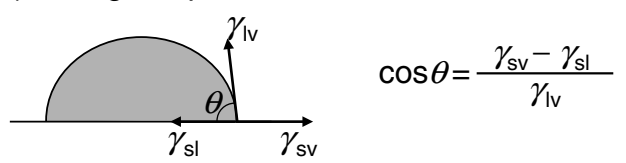

(b) Wenzel's Equation

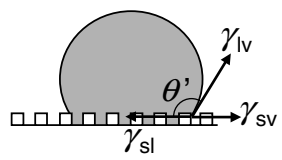

$\cos \theta^{\prime}=r \cos \theta$

$r$ : roughness factor

(c) Cassie's Equation

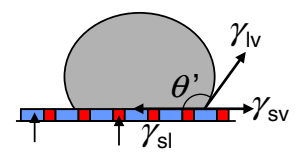

$\cos \theta^{\prime}=f \cos \theta_{1}-(1-f) \cos \theta_{2}$

$f$ : area fraction of region: 1

region:1 region:2

Fig. 3. (Color online) Young, Wenzel, and Cassie models for hydrophobicity of solid surfaces with and without roughness.

$$
\cos \theta^{\prime}=f \cos \theta+(1-f) \cos 180^{\circ}=f \cos \theta+f-1
$$

Figure 3 presents models of Wenzel and Cassie. When the surface roughness is small, Wenzel's mode is dominant. However Cassie's mode becomes dominant with increasing surface roughness. Johnson Jr. and Dettre simulated the contact angle of a water droplet on idealized sinusoidal surfaces, and demonstrated that the dominant mode transition from Wenzel to Cassie occurs when the roughness factor becomes greater than a certain level (ca. 1.7). ${ }^{38)}$ In a practical hydrophobic surface with roughness, both modes will exist. An excellent water shedding property on a superhydrophobic surface (as described later) is attained by increasing the contribution of Cassie's mode, which decreases the practical contact area between the solid and liquid. ${ }^{39)}$ So far, various limitations and corrections have been pointed out and proposed for these two models. ${ }^{40-45)}$ However, as a first approximation, Wenzel's and Cassie's equations are applicable to the relation between solid surface structure and wettability in many cases unless the relative magnitude between surface roughness and droplet size is quite different.

Surfaces, materials, and states whose water contact angles are greater than $150^{\circ}$ are generally designated as superhydrophobic. As described above, such surfaces are not attainable merely by decreasing the surface energy. All superhydrophobic surfaces and coatings have been obtained by combining surface roughness with lower surface energy. ${ }^{46)-50)}$ Although several examples of highly hydrophobic $\left(\mathrm{CA}>120^{\circ}\right)$ surfaces comprising only inorganic materials whose intrinsic surface energy is higher than that of organic compounds were reported, ${ }^{51), 52)}$ these ones should be related to the specific surface shape and adsorption of organic compounds.

Quéré et al. sandwiched a water droplet between two superhydrophobic plates under loading and demonstrated that the water contact angle decreases after de-loading. ${ }^{53)}$ This result implies that Cassie's and Wenzel's modes do not always appear as the most stable state. A similar wetting transition from Cassie to Wenzel was reported experimentally also by evaporation, ${ }^{54)}$ condensation, ${ }^{55)-57)}$ and vibration. ${ }^{58)}$ The stability of these two modes is discussed with consideration of the surface structure and energy balance. ${ }^{59)-61)}$ 
(a)

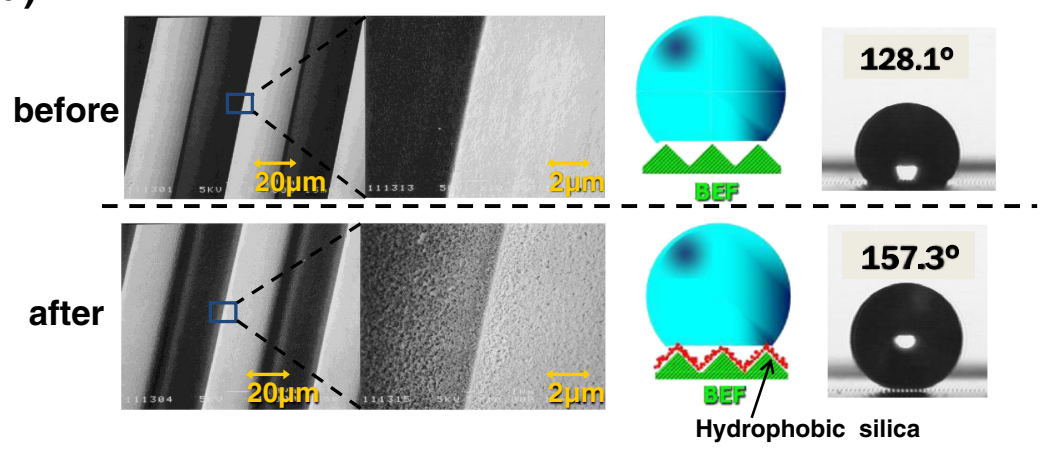

(b)

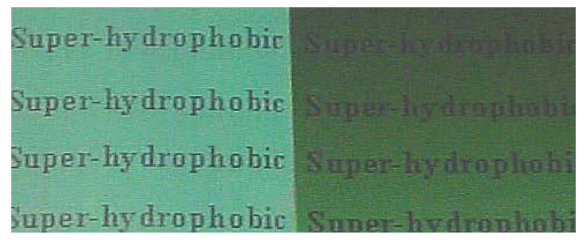

with film without fillm

Fig. 4. (Color online) (a) Surface morphology and hydrophobicity of the Brightness Enhancement Film (BEF) before (upper) and after (lower) coating hydrophobic silica particles. (b) Brightness enhancement property of backlighting of obtained superhydrophobic film (Left: backlighting with the film, Right: backlighting only). The observation angle was about $45^{\circ}$ to the upright.

Recently, various studies of superhydrophobic surface have been conducted in all over the world. Miwa et al. derived an equation which describes the relation between sliding angles and contact angles on superhydrophobic surfaces by assuming continuous circular cone structure as a surface roughness. ${ }^{39}$ ) Sakai et al. prepared various superhydrophobic surfaces using $\mathrm{ZnO}$ nanorod arrays of different diameters. They revealed that the contact angle was equivalent to the calculated value by assuming the topmost surface of the rods as a solid-liquid contact area, and that the sliding acceleration was governed by the solid area fraction. ${ }^{62)}$ The contents of the studies extend from the new processing route for the surface to the droplet behavior on the surface such as sliding, ${ }^{39), 63)-66)}$ freezing, ${ }^{67), 68)}$ evaporation, ${ }^{69)-71)}$ and bouncing. ${ }^{72)}$ However, such surface has not been put into practical use except in very limited cases.

Superhydrophobic surfaces require surface roughness. Therefore, long-time outdoor durability and mechanical strength are difficult to obtain. Surface roughness engenders a place for stain accumulation on the surface; surfaces with a high roughness commonly show poorer abrasion resistance than flat surfaces do. Especially in the case of transparent superhydrophobic surface, surface roughness is expected to be less than $100 \mathrm{~nm}(R \mathrm{a})$ to suppress visible light scattering. Such a fine structure is easily broken by pressure or abrasion. Although addition of a $\mathrm{TiO}_{2}$ photocatalyst, a roughness mixture, and providing conductivity improve the durability of superhydrophobic surfaces against outdoor exposure, ${ }^{73-75)}$ their effects are still insufficient from the requirements for practical use. Natural lotus leaves possess selfrenewal capability by supplying clean wax to the surface by their own metabolism. This function maintains superhydrophobicity of the plant surface for its entire lifetime. Unfortunately, studies to breakthrough these weak points for superhydrophobic surface are not often reported. To make superhydrophobic surfaces a key technology of surface functional materials, development of technologies for the improvement of mechanical strength and long-term outdoor durability are indispensable.

\subsection{Surface topography and roughness alignment} In the Wenzel and Cassie equations, effects of (a) alignment and (b) topography of the roughness on the wettability are ignored. Yoshimitsu et al. demonstrated that the contribution of Cassie's mode to overall hydrophobicity is increased when the surface has a large area into which water has difficulty penetrating (such as the bottom edge of a rectangular hydrophobic channel). ${ }^{66)}$ They also demonstrated that a proper design of the surface with respect to shape and extent of the three phase line is more effective than the increase of contact angles merely by decreasing the solid-water contact area. The effects of length and direction of three-phase contact line is more significant to the sliding angle than the contact angle. Hydrophobicity of the solid is enhanced by surface roughness. However, the degree depends on the surface shape. Therefore, an additional function can be provided to the hydrophobic surface by designing a roughness shape. Sasaki and Takeda reported the preparation of transparent films with both superhydrophobicity and brightness enhancement property (the brightness of light transmitted can be enhanced in a specific direction) by using a film with specific surface topography. ${ }^{75), 76)}$ They prepared a coating liquid by mixing solvents, fluoro-acrylic resin, and commercial hydrophobic silica particles whose size was much smaller than the wavelength of visible light. Then, they coated it on the film (brightness enhancement film; BEF) to provide superhydrophobicity by combining the roughness of the BEF with that of the silica particles (Fig. 4). Detailed analysis on this film based on surface topography suggests that water droplets are supported at the topmost portion of the film surface. ${ }^{77)}$ Combination of roughness with different shape and dimension to solid with a low surface energy increase both hydrophobicity and durability. ${ }^{78)-80)}$ Theoretical analysis on this effect based on surface structure has also been carried out. ${ }^{77)}$

The degree of contribution of roughness or chemical composition in the hydrophobic surfaces to contact angle, sliding angle, and sliding acceleration is different. Therefore, precise control of 
the surface composition and its structure is required for the preparation of surface which satisfies static and dynamic hydrophobicity simultaneously. Proper design of surface structure and chemical composition enables control of directional transportation and sliding of a liquid droplet.

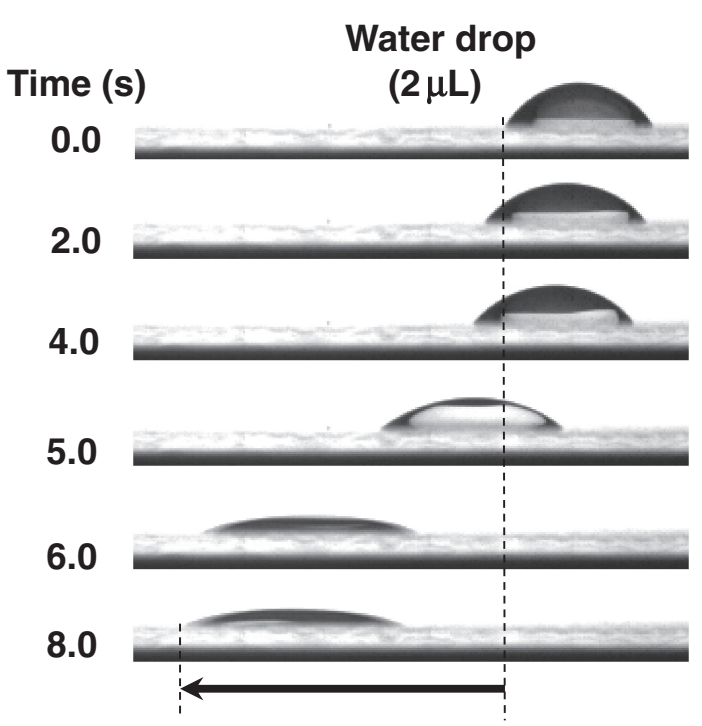

Fig. 5. Water drop movement on a surface with a chemical composition gradient. The surface was prepared by moving sample stage with the controlled velocity when the ODS ( $n$-octadecyltrimethoxysilane) coated glass plate was irradiated by VUV light through a photomask. The dotted lines are the initial and final positions of advancing points for the droplet placed on the gradient surface. Water drop moved from hydrophobic-side (right) to hydrophilic-side (left) on the surface.

\section{Motion of water droplet on hydrophobic surface}

\subsection{Droplet motion by controlling solid surface}

Chaudury et al. demonstrated that a water droplet moves uphill by providing merely a spatial gradient to the decyltrichlorosilane. ${ }^{81)}$ Using that method, a liquid droplet is controllable by providing a gradient in solid surface characteristics such as shape ${ }^{82)}$ roughness, ${ }^{83), 84)}$ surface energy ${ }^{81), 85)}$ (Fig. 5), and their mixture with external field. ${ }^{86}$ In these surfaces, a Laplacian pressure difference is generated between advancing and receding sides of the liquid: the liquid droplets move spontaneously.

Micropatterned surfaces with different wettability are well known to provide wetting anisotropy. ${ }^{24}$ The degree of that anisotropy depends on the line structure (line width), chemical composition, and its homogeneity. Suzuki et al. prepared a line-patterned surface using two silane coupling agents, then demonstrated that selection of droplets is feasible by setting the surface with rotating in-plane on the slope surface ${ }^{87)}$ (Fig. 6). This result is explained by the balance between gravitational and retentive forces. Directional transportation of a water droplet on a superhydrophobic surface with hydrophilic path line was also demonstrated experimentally by $\mathrm{Xu}$ et al. ${ }^{88)}$ Similar sliding anisotropy was obtainable by preparing a line-patterned groove structure on a hydrophobic surface. ${ }^{89)}$

The internal fluidity of a water droplet sliding between two parallel plates on a slope was observed directly using PIV method with a high-speed camera system. The interfacial caterpillar-like rotation flows with slip motion at the solid-liquid interface were related to the combination of top and bottom wettabilities. ${ }^{90)}$ A droplet's shape, solid-liquid molecular interaction, and the gravitic force will be important factors determining the sliding mode on a solid surface. Control of liquid droplets on solid (a)

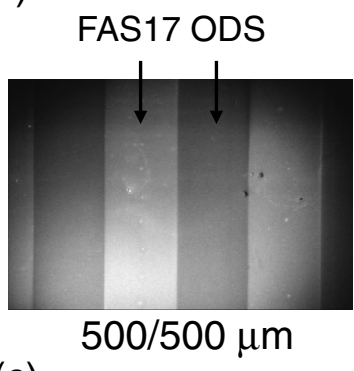

(c)
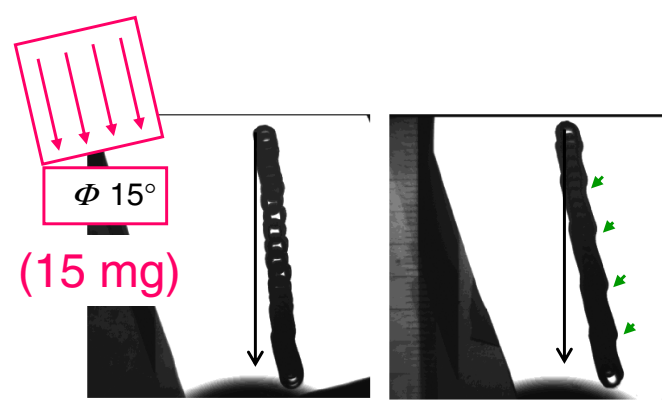

(b)

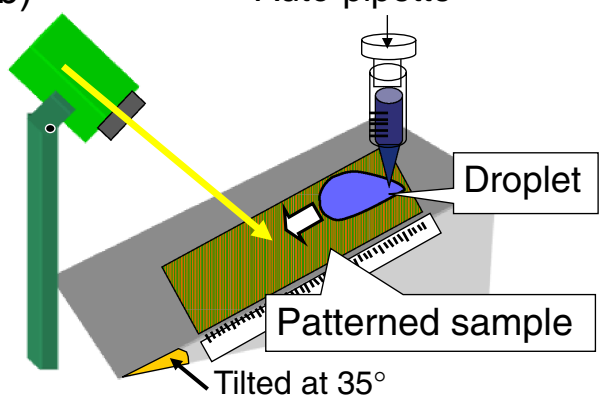

sake(49 $\left.\mathrm{mJ} \mathrm{m}^{-2}\right)$ shochu(42 $\left.\mathrm{mJ} \mathrm{m}^{-2}\right)$ awamori(30 $\left.\mathrm{mJ} \mathrm{m}^{-2}\right)$ whiskey $\left(28 \mathrm{~mJ} \mathrm{~m}^{-2}\right)$

Fig. 6. (Color online) (a) FE-SEM micrographs of the fluoroalkylsilane surface periodically line-patterned by octadecyltrimethoxysilane region with line/space of $500 / 500 \mu \mathrm{m}$, (b) experimental setup for the evaluation of water droplets' sliding behavior on these surface from top view, and (c) sliding behavior of droplets with various alcoholic beverages on a $500 \mu \mathrm{m}$ linesurface rotated at $\Phi=15^{\circ}$ on a slope tilted at $35^{\circ}$. The image-recording interval is $200 \mathrm{~ms}$. The displacement angle depends on the liquid qaulity. The number described in the bracket is surface energy of each liquid. 

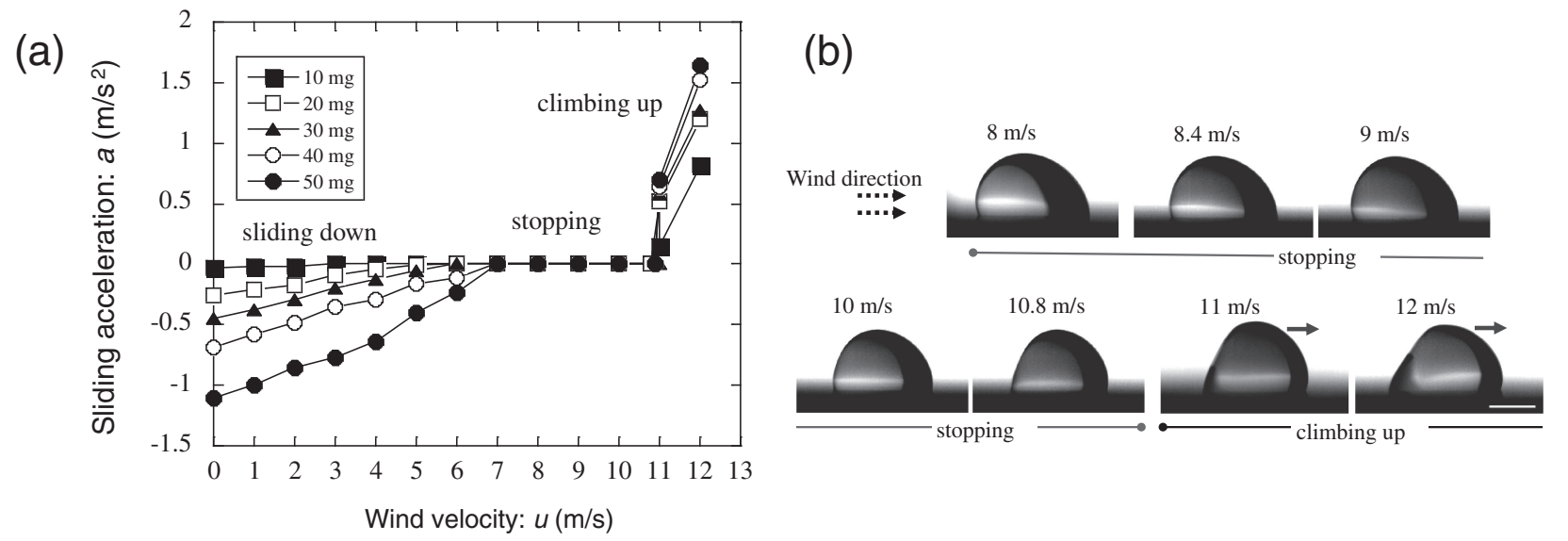

Fig. 7. (a) Variations of the sliding and climbing acceleration of $10-50 \mathrm{mg}$ water droplets on the $35^{\circ}$-tilted octadecyltrimethoxysilane-coated surface as a function of the wind velocity. (b) Change of the morphology for $50 \mathrm{mg}$ water droplets on the surface in air flowing with different wind velocity during stopping and climbing regions. The camera was tilted with the surface, therefore droplet moves from right to left when sliding. The droplet stopped when wind velocity is small, changing its shape from a forward-bent to backward-bent posture against the surface inclination, then it climbed along the inclined surface.

surfaces has attracted much attention as an indispensable technology for use with various industrial items including MEMS or lab-on-a-chip, especially for hydrophobic coatings.

\subsection{Droplet motion using external field}

To date, various investigations have been conducted to examine the motion of a liquid droplet on a solid surface using external fields such as those of gravity, a heat gradient, ${ }^{91), 92)}$ electric fields, ${ }^{93)-95)}$ magnetic fields, ${ }^{96-98)}$ photo-illumination, ${ }^{99)}$ blowing wind, ${ }^{100)}$ and vibration. ${ }^{101)}$

Ichimura et al. successfully provided macroscopic motion to liquids on a flat solid surface by photo irradiation to monolayer covering the surface. ${ }^{99)}$ In their research, photoisomerization of surface azobenzenes leads to contact angle hysteresis and consequent directional motion. Hashimoto et al. investigated the relation between airflow and the motion of a water droplet on an inclined hydrophobic coating using a high-speed camera system and an open-circuit-type wind tunnel. ${ }^{100)}$ They reported that the motions of a water droplet in an air stream are classifiable into three: sliding, stopping, and climbing (Fig. 7), and that the wind velocity dependence differs between sliding and climbing acceleration.

The motion on the normal hydrophobic surface is not always fast, which sometimes constitutes a barrier against application of surface wettability to industrial devices. A superhydrophobic surface enables rapid transportation of water droplets. Various systems have been proposed by combining this surface and external fields. Takeda et al. controlled water droplet motion of a superhydrophobic surface using an electric field. In their system, the Coulomb force of the electric charge provided to the water from the solid surface was the dominant driving force for the water droplet movement. ${ }^{93), 95)}$ Recently, internal fluidity of water droplets during transportation on superhydrophobic surface by an external electric field is also analyzed using a PIV system, revealing that the droplet motion is slipping and that dielectrophoretic force plays an important role in movement by an electric field. ${ }^{102)}$ Surface wettability control by using external electric field is often called as electrowetting. This technology grows explosively as a driving mechanism for a various fluidic and electro-optic applications. ${ }^{103)-107)}$

\section{Summary}

So far, studies of hydrophobic surface have mainly examined the relation between the structure or chemical composition of a solid surface, and the contact angle or sliding angle. These are fundamentally examples of static hydrophobicity, and provide no information related to dynamic hydrophobicity. Detailed investigations of the effects of solid composition, surface structure, their alignment, and their homogeneity on dynamic hydrophobicity are necessary for the design of surfaces with excellent water-shedding properties.

Behaviors of liquids have been treated mainly by fluid mechanics and physical chemistry of the solid-liquid interface. However, for better understanding of the relation between solid surface characters and dynamic hydrophobicity, the viewpoint of materials science is important. The author believes interdisciplinary studies in these three academic fields are necessary. Collaborative studies for dynamic hydrophobicity might establish a new academic field situated on the border separating these three sciences.

Liquids and gels possess greater deformation flexibility than solids do. The control of liquids is a key technology for the new functional surfaces or devices. This technology also contributes to reduction of greenhouse gas emissions through the decrease of friction drag, thereby conserving energy. Basic research related to the control of liquid droplets on solid materials remains insufficient. Because this is a vital technology for our future, further investigations are expected in this field.

\section{References}

1) A. Nakajima, "Kotai Hyoumen no Nure Seigyo", Uchida Rokakuho Press, Tokyo, Japan (2007) pp. 29-39 [in Japanese].

2) A. Nakajima, K. Hashimoto and T. Watanabe, Hikari Kagaku, 30, 199-206 (1999) [in Japanese].

3) T. Nishino, M. Meguro, K. Nakamae, M. Matsushita and Y. Ueda, Langmuir, 15, 4321-4323 (1999).

4) J. Drelich and J. D. Miller, J. Colloid Interface Sci., 164, $252-$ 259 (1994).

5) A. Amirfazli, D. Y. Kwok, J. Gaydos and A. W. Neumann, J. Colloid Interface Sci., 205, 1-11 (1998). 
6) A. Amirfazli, S. Hänig, A. Müller and A. W. Neumann, Langmuir, 16, 2024-2031 (2000).

7) J. Drelich, Colloids Surf., A, 116, 43-54 (1996).

8) T. Furuta, A. Nakajima, M. Sakai, T. Isobe, Y. Kameshima and K. Okada, Chem. Lett., 38, 580-581 (2009).

9) T. Furuta, M. Sakai, T. Isobe and A. Nakajima, Chem. Lett., 38, 1092-1093 (2009).

10) T. Furuta, M. Sakai, T. Isobe and A. Nakajima, Langmuir, 25, 11998-12001 (2009).

11) C. G. L. Furmidge, J. Colloid Sci., 17, 309-324 (1962).

12) E. B. Dussan V., J. Fluid Mech., 151, 1-20 (1985).

13) A. Carre and M. E. R. Shanahan, J. Adhes., 49, 177-185 (1995).

14) M. Sakai, J.-H. Song, N. Yoshida, S. Suzuki, Y. Kameshima and A. Nakajima, Surf. Sci., 600, L204-L208 (2006).

15) S. Gogte, P. Vorobieff, R. Truesdell, A. Mammoli, F. van Swol, P. Shah and C. J. Brinker, Phys. Fluids, 17, 51701 (2005).

16) S. R. Hodges, O. E. Jensen and J. M. Rallison, J. Fluid Mech., 512, 95-131 (2004).

17) A. Nakajima, S. Suzuki, Y. Kameshima, N. Yoshida, T. Watanabe and K. Okada, Chem. Lett., 32, 1148-1149 (2003).

18) M. Sakai, J.-H. Song, N. Yoshida, S. Suzuki, Y. Kameshima and A. Nakajima, Langmuir, 22, 4906-4909 (2006).

19) M. Sakai, A. Hashimoto, N. Yoshida, S. Suzuki, Y. Kameshima and A. Nakajima, Rev. Sci. Instrum., 78, 045103 (2007).

20) S. Suzuki, A. Nakajima, Y. Sakurada, M. Sakai, N. Yoshida, A. Hashimoto, Y. Kameshima and K. Okada, J. Jpn. Soc. Colour Mater., 82, 3-8 (2009).

21) Y. Sakurada, S. Suzuki, M. Sakai, A. Nakajima, Y. Kameshima and K. Okada, Chem. Lett., 37, 688-689 (2008).

22) "Research Report of Superhydrophobic/philic Project", Kanagawa Academy of Science and Technology, Kanagawa, Japan (2007) pp. 1-75 [in Japanese].

23) N. Yoshida, Y. Abe, H. Shigeta, A. Nakajima, H. Ohsaki, K. Hashimoto and T. Watanabe, J. Am. Chem. Soc., 128, 743747 (2006).

24) M. Morita, T. Koga, H. Otsuka and A. Takahara, Langmuir, 21, 911-918 (2005).

25) T. Kako, A. Nakajima, H. Irie, Z. Kato, K. Uematsu, T. Watanabe and K. Hashimoto, J. Mater. Sci., 39, 547-555 (2004).

26) J.-H. Song, M. Sakai, N. Yoshida, S. Suzuki, Y. Kameshima and A. Nakajima, Surf. Sci., 600, 2711-2717 (2006).

27) T. Furuta, M. Sakai, T. Isobe, S. Matsushita and A. Nakajima, Langmuir, 27, 7307-7313 (2011).

28) S. Suzuki, M. Sakai, N. Yoshida, A. Hashimoto, Y. Kameshima, K. Okada and A. Nakajima, J. Jpn. Soc. Colour Mater., 83, 499-504 (2010).

29) S. Suzuki, A. Nakajima, M. Sakai, Y. Sakurada, N. Yoshida, A. Hashimoto, Y. Kameshima and K. Okada, Chem. Lett., 37, 58-59 (2008).

30) S. Suzuki, A. Nakajima, N. Yoshida, M. Sakai, A. Hashimoto, Y. Kameshima and K. Okada, Chem. Phys. Lett., 444, 37-41 (2007).

31) S. Suzuki, A. Nakajima, N. Yoshida, M. Sakai, A. Hashimoto, Y. Kameshima and K. Okada, Langmuir, 23, 8674-8677 (2007).

32) T. Furuta, A. Nakajima, M. Sakai, T. Isobe, Y. Kameshima and K. Okada, Langmuir, 25, 5417-5420 (2009).

33) Y. L. Chen, C. A. Helm and J. N. Israelachvili, J. Phys. Chem., 95, 10736-10747 (1991).

34) L. Gao and T. J. McCarthy, Langmuir, 23, 3762-3765 (2007).

35) A. Marmur and E. Bittoun, Langmuir, 25, 1277-1281 (2009).

36) R. N. Wenzel, Ind. Eng. Chem., 28, 988-994 (1936).

37) A. B. D. Cassie and S. Baxter, Trans. Faraday Soc., 40, 546551 (1944).

38) R. E. Johnson, Jr. and R. H. Dettre, Adv. Chem. Ser., 43, 112-
135 (1963).

39) M. Miwa, A. Nakajima, A. Fujishima, K. Hashimoto and T. Watanabe, Langmuir, 16, 5754-5760 (2000).

40) J. Drelich, J. D. Miller, A. Kumar and G. M. Whitesides, Colloids Surf., A, 93, 1-13 (1994).

41) J. T. Woodward, H. Gwin and D. K. Schwartz, Langmuir, 16, 2957-2961 (2000).

42) N. A. Patankar, Langmuir, 19, 1249-1253 (2003).

43) A. Marmur, Langmuir, 19, 8343-8348 (2003).

44) J. Long, M. N. Hyder, R. Y. M. Huang and P. Chen, $A d v$. Colloid Interface Sci., 118, 173-190 (2005).

45) M. Nosonovsky, Langmuir, 23, 9919-9920 (2007).

46) A. Nakajima, K. Hashimoto and T. Watanabe, Monatsh. Chem., 132, 31-41 (2001).

47) X. Feng and L. Jiang, Adv. Mater., 18, 3063-3078 (2006).

48) R. Blossey, Nat. Mater., 2, 301-306 (2003).

49) M. Ma and R. M. Hill, Curr. Opin. Colloid Interface Sci., 11, 193-202 (2006).

50) A. Nakajima, J. Ceram. Soc. Japan, 112, 533-540 (2004).

51) X. J. Feng, J. Zhai and L. Jiang, Angew. Chem., Int. Ed., 44, 5115-5118 (2005).

52) M. Li, J. Zhau, H. Liu, Y. L. Song, L. Jiang and D. B. Zhu, J. Phys. Chem. B, 107, 9954-9957 (2003).

53) A. Lafuma and D. Quéré, Nat. Mater., 2, 457-460 (2003).

54) M. Nosonovsky and B. Bhushan, Nano Lett., 7, 2633-2637 (2007).

55) K. A. Wier and T. J. McCarthy, Langmuir, 22, 2433-2436 (2006).

56) C. Dorrer and J. Rühe, Langmuir, 23, 3820-3824 (2007).

57) T. Furuta, M. Sakai, T. Isobe and A. Nakajima, Langmuir, 26, 13305-13309 (2010).

58) E. Bormashenko, R. Pogreb, G. Whyman and M. Erlich, Langmuir, 23, 6501-6503 (2007).

59) L. Barbieri, E. Wagner and P. Hoffmann, Langmuir, 23, 1723-1734 (2007).

60) C. Ishino, K. Okumura and D. Quéré, Europhys. Lett., 68, 419-425 (2004).

61) N. A. Patankar, Langmuir, 20, 7097-7102 (2004).

62) M. Sakai, H. Kono, A. Nakajima, X. Zhang, H. Sakai, M. Abe and A. Fujishima, Langmuir, 25, 14182-14186 (2009).

63) J. P. Youngblood and T. J. McCarthy, Macromolecules, 21, 6800-6806 (1999).

64) D. Öner and T. J. McCarthy, Langmuir, 16, 7777-7782 (2000).

65) D. Richard and D. Quéré, Europhys. Lett., 48, 286-291 (1999).

66) Z. Yoshimitsu, A. Nakajima, T. Watanabe and K. Hashimoto, Langmuir, 18, 5818-5822 (2002).

67) P. Tourkine, M. L. Merrer and D. Quéré, Langmuir, 25, 7214 7216 (2009).

68) H. Wang, L. M. Tang, X. M. Wu, W. T. Dai and Y. P. Qiu, Appl. Surf. Sci., 253, 8818-8824 (2007).

69) K. A. Wier and T. J. McCarthy, Langmuir, 22, 2433-2436 (2006).

70) G. McHale, S. Aquil, N. J. Shirtcliffe, M. I. Newton and H. Y. Erbil, Langmuir, 21, 11053-11060 (2005).

71) X. Y. Zhang, S. X. Tan, N. Zhao, X. L. Guo, X. L. Zhang and Y. J. Zhang, ChemPhysChem, 7, 2067-2070 (2006).

72) D. Bartolo, F. Bouamrirene, E. Verneuil, A. Buguin, P. Siberzan and S. Moulinet, Europhys. Lett., 74, 299-305 (2006).

73) A. Nakajima, K. Hashimoto, T. Watanabe, K. Takai, G. Yamauchi and A. Fujishima, Langmuir, 16, 7044-7047 (2000).

74) A. Nakajima, K. Abe, K. Hashimoto and T. Watanabe, Thin Solid Films, 376, 140-143 (2000).

75) M. Sasaki, N. Kieda, K. Katayama, K. Takeda and A. Nakajima, J. Mater. Sci., 39, 3717-3722 (2004).

76) K. Takeda, M. Sasaki, N. Kieda, K. Katayama, T. Kako, K. 
Hashimoto, T. Watanabe and A. Nakajima, J. Mater. Sci. Lett., 20, 2131-2133 (2001).

77) Z. Yoshimitsu, A. Nakajima, T. Watanabe and K. Hashimoto, J. Surf. Finish. Soc. Jpn., 56, 925-929 (2005) [in Japanese].

78) A. Nakajima, Z. Yoshimitsu, C. Saiki, K. Hashimoto and T. Watanabe, "Ceramic Processing Science IV", Ceramic Transactions vol. 112, Ed. by S. Hirano, G. L. Messing and N. Claussen, American Ceramic Society, Westerville, Ohio, U.S.A. (2001) p. 323-328.

79) K. Takeda, T. Kuramochi, T. Nozawa, N. Kieda, K. Katayama and A. Nakajima, J. Surf. Finish. Soc. Jpn., 54, 677-682 (2003) [in Japanese].

80) T. Yanagisawa, A. Nakajima, Y. Kameshima, M. Sakai and K. Okada, Mater. Sci. Eng., B, 161, 36-39 (2009).

81) M. Chaudury and G. M. Whitesides, Science, 256, 15391541 (1992).

82) J. Zhang and Y. Han, Langmuir, 23, 6136-6141 (2007).

83) G. Fang, W. Li, X. Wang and G. Qiao, Langmuir, 24, 1165111660 (2008).

84) J.-T. Yang, J. C. Chen, K.-J. Huang and J. A. Yeh, J. Microelectromech. Syst., 15, 697-707 (2006).

85) Y. Ito, M. Heydari, A. Hashimoto, T. Konno, A. Hirasawa, S. Hori, K. Kurita and A. Nakajima, Langmuir, 23, 1845-1850 (2007).

86) A. Shastry, M. J. Case and K. F. Böhringer, Langmuir, 22, 6161-6167 (2006).

87) S. Suzuki, A. Nakajima, K. Tanaka, M. Sakai, A. Hashimoto, N. Yoshida, Y. Kameshima and K. Okada, Appl. Surf. Sci., 254, 1797-1805 (2008).

88) Q. F. Xu, J. N. Wang, I. H. Smith and K. D. Sanderson, Appl. Phys. Lett., 93, 233112 (2008).

89) A. D. Sommers and A. M. Jacobi, J. Micromech. Microeng., 16, 1571-1578 (2006).

90) S. Suzuki, A. Nakajima, M. Sakai, A. Hashimoto, N. Yoshida, Y. Kameshima and K. Okada, Appl. Surf. Sci., 255, 34143420 (2008).
91) J. B. Brzoska, F. Brochard and F. Rondelez, Langmuir, 9, 2220-2224 (1993).

92) V. Pratap, N. Moumen and R. S. Subramanian, Langmuir, 24, 55185-55193 (2008).

93) K. Takeda, A. Nakajima, K. Hashimoto and T. Watanabe, Jpn. J. Appl. Phys., 41, 287-291 (2002).

94) M. Gunji and M. Washizu, J. Phys. D: Appl. Phys., 38, 24172423 (2005).

95) K. Takeda, A. Nakajima, K. Hashimoto and T. Watanabe, Surf. Sci., 519, 589-592 (2002).

96) X. Hong, X. Gao and L. Jiang, J. Am. Chem. Soc., 129, 14781479 (2007).

97) S. Afkhami, Y. Renardy, M. Renardy, J. S. Riffle and T. StPierre, J. Fluid Mech., 610, 363-380 (2008).

98) H. Asakura, A. Nakajima, M. Sakai, S. Suzuki, Y. Kameshima and K. Okada, Appl. Surf. Sci., 253, 3098-3102 (2007).

99) K. Ichimura, S.-K. Oh and M. Nakagawa, Science, 288, 1624-1626 (2000).

100) A. Hashimoto, M. Sakai, N. Yoshida, S. Suzuki, Y. Kameshima and A. Nakajima, J. Surf. Finish. Soc. Jpn., 59, 907-912 (2008).

101) L. Dong, A. Chaudhury and M. K. Chaudhury, Eur. Phys. J. E, 21, 231-242 (2006).

102) M. Sakai, H. Kono, A. Nakajima, H. Sakai, M. Abe and A. Fujishima, Langmuir, 26, 1493-1495 (2010).

103) M. G. Pollack, A. D. Shenderov and R. B. Fair, Lab Chip, 2, 96-101 (2002).

104) S. K. Cho, H. Moon and C.-J. Kim, J. Microelectromech. Syst., 12, 70-80 (2003).

105) V. Srinivasan, V. K. Pamula and R. B. Fair, Anal. Chim. Acta, 507, 145-150 (2004).

106) L. Dong, A. K. Agarwal, D. J. Beebe and H. Jiang, Nature, 442, 551-554 (2006).

107) V. Hessel, H. Lowe and F. Schonfeld, Chem. Eng. Sci., 60, 2479-2501 (2005).

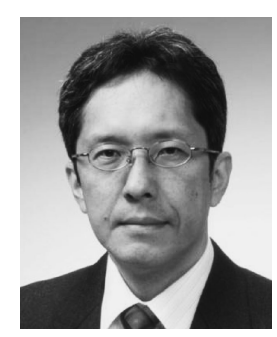

Akira Nakajima earned his B.E. and M.E. degrees in Inorganic Materials Science from Tokyo Institute of Technology in 1985 and 1987, respectively. He then joined Japan Energy Corp. as a research scientist. In 1997, he earned his Ph.D. from The Pennsylvania State University. He moved to RCAST of The University of Tokyo in 1998 and began research on hydrophobic coatings. He established a venture company (ASTI: Advanced Systems of Technology Incubation) and became its CTO in 2001. He joined the faculty staff of the Tokyo Institute of Technology in March 2003. Currently, he is a Professor of the Department of Metallurgy and Ceramic Science. He was the leader of the "Super Hydrophilic/phobic Surface Project" at the Kanagawa Academy of Science and Technology (KAST) during 2003-2007. 